Bull. Mater. Sci., Vol. 6, No. 2, May 1984, pp. 129-149. (C) Printed in India.

\title{
Sophisticated equipment developed for growth and evaluation of perfection of nearly perfect crystals
}

\author{
KRISHAN LAL and AJIT RAM VERMA \\ National Physical Laboratory, Hillside Road, New Delhi 110012, India
}

\begin{abstract}
This paper reviews four major equipment developed at the National Physical Laboratory for growth and perfection evaluation of singie crystals, namely (i) a crystal puller for growth of nearly perfect crystals by the Czochralski method; (ii) a microfocus $x$-ray generator; (iii) an $\mathrm{x}$-ray diffraction topography camera; and (iv) a triple crystal $\mathrm{x}$-ray diffractometer. The crystal puller can provide smooth, uniform and variable pulling rates. The maximum length of pull is nearly $60 \mathrm{~cm}$. Efforts have been made to isolate vibrations. Nearly perfect single crystals of $\mathrm{KCl}, \mathrm{KBr}$ and $\mathrm{NaCl}$ with maximum diameter of $\sim 60 \mathrm{~mm}$ have been grown. The crystals give diffraction curves with half width in the range of 10-30 sec of arc. In the projection topographs, dislocations can be resolved and characterized. The microfocus $x-$ ray generator is a demountable continuously evacuated system with specially designed electron gun and anode assembly. The vacuum is continuously monitored for ease of maintenance. In the point focus mode the spot size is $40 \mu \mathrm{m}$ on the anode. X-ray topography system is a versatile equipment used for projection and section topography. It can provide $360^{\circ}$ rotations to the specimen disc around an axis perpendicular to it. Rotations of a few sec of arc can be given to the specimen around a vertical axis. Typical diffraction curves of a dislocation-free crystal and a crystal with boundaries are shown. Well-resolved images of dislocations are shown in a topograph as an illustration. In the triple crystal $x$-ray diffractometer a highly collimated and monochromated $\mathrm{K} \alpha_{1}$ exploring $\mathrm{x}$-ray beam is obtained by combining microfocus source, $\mathrm{a}$ special collimator and crystal monochromators of Bonse-Hart type. With this beam very narrow diffraction curves with half width of about $5 \mathrm{sec}$ of arc can be recorded. Typical results of measurement of diffuse x-ray scattering (DXs) on nearly perfect silicon single crystals are discussed. It has been observed that the contribution of phonons to the DXs is negligible. The DXS is mainly due to point defect aggregates.
\end{abstract}

Keywords. Crystal puller; microfocus $\mathrm{x}$-ray generator; $\mathrm{x}$-ray diffraction topography camera; $\mathrm{x}$-ray diffractometer.

\section{Introduction}

Development of modern science and technology is to a great extent the result of very strong interaction between the experimentalists and the theoreticians. Advances in techniques and equipment have played a vital role in the growth of experimental sciences. Development of better techniques and equipment not only leads to a deep and precise understanding of physical phenomenon but it also leads to discovery of new science and technology.

The role of technique and equipment development in the growth of science and technology in the industrially less-developed countries is all the more important as the infrastructure is weak in these countries. It is, therefore, for the national research institutions to play a leading role in this direction.

The National Physical Laboratory had been set up primarily for the maintenance of standards of units of measurements. Work on some industrially important materials like carbons, piezoelectric ceramics and semiconducting materials was also pursued. 
The reproducible preparation of these materials with desired properties required considerable effort in setting up of infrastructure for preparation and characterization of materials. Facilities were set up for characterization of materials regarding chemical composition, crystalline structure and crystalline perfection. Soon it was realized that for the activities in the material science area as well as in the standards measurements, the characterization of materials is to play an important role. Without having a feeling for the relationship of the properties of a material with its chemical composition and real crystalline structure (i.e. structure and defects), no real progress can be made. Further, the role of crystal growth as an activity occupying central position in material science as well as solid state physics was identified.

During the last nearly fifteen years, a determined effort was made at the National Physical Laboratory to develop a nucleus for design, development and fabrication of sophisticated equipment for growth and characterization of nearly perfect crystals regarding perfection. Attempt was made to develop those techniques and equipment which will take the subject to the state of art level. A number of techniques and equipment have been successfully developed. Important among the techniques are:

(i) a technique for growth of nearly perfect single crystals from the melt by the Czochralski method (Krishan Lal et al 1982; Verma 1982),

(ii) $\mathrm{x}$-ray diffraction topographic methods for characterization of single crystals regarding perfection (Krishan Lal 1981, 1982),

(iii) a high resolution diffuse $x$-ray scattering techniques for study of point defect aggregates in nearly perfect single crystals (Krishan Lal and Singh 1977; Krishan Lal 1980; 1982),

(iv) a high resolution $\mathrm{x}$-ray diffraction method for direct observation and characterization of microstructural changes induced by high electric fields in single crystals of insulators and semiconductors (Krishan Lal and Peter Thoma 1981a, b),

(v) a high resolution $\mathrm{x}$-ray Laue method for study of lattice imperfections in whisker crystals (Krishan Lal and Peneva 1968, 1969),

(vi) a technique for precise measurement of dielectric constant of solid insulators at low frequencies (Krishan Lal and Jhans 1977), and

(vii) a technique for measurement of dielectric loss and electrical conductivity of insulating crystals under controlled temperature and environment (Krishan Lal and Pahwa 1971).

Following are thè major equipment that have been developed as a result of this effort:

(i) microfocus $\mathrm{x}$-ray generators as sources for high resolution $\mathrm{x}$-ray diffraction work (Krishan Lal et al 1975),

(ii) X-ray diffraction topography cameras similar to Lang camera (Verma et al 1974),

(iii) triple crystal $\mathrm{x}$-ray diffractometers for recording diffraction curves (rocking curves), high resolution topographs and for measurement of diffuse x-ray scattering from nearly perfect single crystals at high resolution (Krishan Lal and Singh 1977; Krishan Lal 1980, 1982),

(iv) a triple and a quadruple crystal $x$-ray diffractometers for study of electric field induced microstructural changes in semiconductor and insulator single crystals (Krishan Lal and Peter Thoma 1981a, b),

(v) crystal pulling equipment for growth of nearly perfect single crystals from the melt by the Czochralski method (Krishan Lal et al 1979). 
A number of research investigations have been carried out successfully using the techniques and the equipment listed above. These have yielded results of considerable significance (Peneva 1969; Krishan Lal et al 1970, 1979; Krishan Lal and Singh 1980; Singh 1980; Krishan Lal and Kumar 1978; Krishan Lal 1980, 1982; Krishan Lal et al 1982; Thoma and Krishan Lal 1982; Krishan Lal and Thoma 1982; Kumar 1983).

In the present paper, we shall briefly describe the salient features of the major equipment developed for crystal growth and characterization of single crystals regarding perfection. Some important results obtained with these equipment will also be briefly described.

\section{Equipment for growth of nearly perfect crystals from melt}

\subsection{Basic principles}

A single crystal by definition is a three-dimensional periodic arrangement of the constituent atoms, ions or molecules in space. In other words, the single crystal has a short and a long range order. However, it is not possible to realize an ideal crystal with a perfect periodic arrangement of constituent units. All real crystals have regions where the periodic arrangement is disturbed by 'lattice imperfections' or 'crystal defects'. The main lattice imperfections are: (i) grain boundaries; (ii) small angle boundaries (iii) dislocations; (iv) impurities and their aggregates; and (v) vacancies and their clusters. A crystal can be considered as nearly perfect when it is free from boundaries and density of its dislocations is less than $10^{5} / \mathrm{cm}^{2}$.

For the present-day scientific and technological applications, nearly perfect crystals with controlled chemical composition are required. A number of techniques have been developed for preparation of such crystals. The starting material is a powder or polycrystalline mass. The transformation from one solid (polycrystalline) to the other solid phase (single crystal) is achieved by first converting the starting material into a vapour or a liquid phase (melt). In some extreme situations a solid-to-solid transformation is also used. The intermediate phase is converted into the final solid phase in a controlled manner, so that the product has a nearly perfect ordering of constituents. During this process protection is to be provided against chemical contamination.

The intense research and development work of the last few decades has shown that the techniques employing growth of șingle crystals from the melt are best suited for economic production of large quantities of nearly perfect single crystals having controlled chemical composition. However, inspite of its extensive applications this method cannot be considered to be a general method. It can be used only if the solid and the liquid phases of the material are in thermodynamical equilibrium with each other near the melting point.

Important methods used for growth of single crystals from the melt are (i) Kyropoulos method, (ii) Bridgman method, (iii) Czochralski method and (iv) Stepanov method or the shaped crystal method.

Among these, the Czochralski method has been most widely used. We shall consider only this method for further discussion.

Figure 1 shows a schematic line diagram depicting the basic features of the Czochralski method. The starting material $M$ is heated in a crucible with the help of a suitable furnace $F$ and the melt is held close to its melting point. A seed crystal $S$ held in 


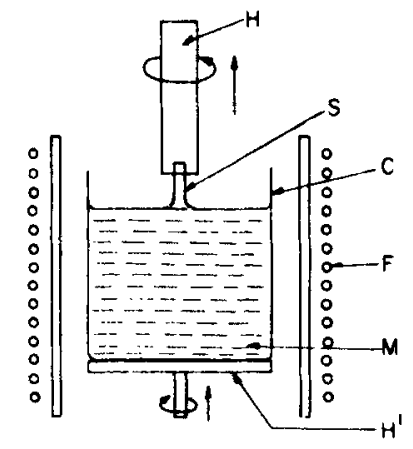

Figure 1. A schematic line diagram showing the basic principle of the techniques used for growth of single crystals from the melt

a suitable seed-holder $H$ is dipped into the melt. The thermal conditions are adjusted in such a manner that the seed and the melt are in contact and in thermal equilibrium. The seed is slowly withdrawn or pulled and the crystal is formed due to freezing of the molten material. The amount of heat supplied to the melt is decreased gradually which leads to a gradual increase in the lateral dimensions of the crystal. The lateral dimensions can also be controlled by changing the rate of pulling. A decrease in the rate of pulling produces an increase in the diameter of the crystal. After having achieved a predetermined size of the crystal, the power fed to the melt and the rate of pulling are controlled in such a way that the diameter does not change. To avoid strains in the crystal due to small variations in the temperature across its diameter, the seed holder is rotated. When crystals with impurity doping are to be grown the uniform distribution of impurity in the melt and the crystal is an additional requirement. The crucible is also rotated but in opposite direction. As the crystal grows, the melt is depleted and its level falls down. To achieve a very strict temperature control the crucible is lifted at a rate which compensates for the lowering in the level of the melt.

The growth of good quality crystals requires strict control on: (i) quality of the seed crystal; (ii) the crucible and the seed-holder materials; (iii) the ambient atmosphere; (iv) the thermal system; and (v) the mechanical system. The seed crystal should be nearly perfect and free of undesirable impurities. In the process of attaining the temperature close to the melting temperature, the crystal should not be subjected to thermal shocks. The processes like necking are used to eliminate the dislocations originating from the seed into the crystal. Also, by increasing the speed of pulling to rates higher than that of the propagation of dislocations, in the initial stage, the dislocations growing from the seed are stopped from being incorporated into the main body of the crystal.

The materials of the seed holder and crucible should not react with the crystal and should be able to stand the temperature. Also, these should not react with the ambient atmosphere. The control of ambient atmosphere is necessary to avoid chemical contamination of the melt and the crystal and also for controlled heat dissipation. In some case the compounds decompose at melting point, under normal conditions. Sometimes decomposition can be avoided by heating the starting material under vapours of one of the constituents at high pressures.

The control of thermal system is very vital for growth of high quality crystals. The furnace is the main source of heat. Heat is transferred by conduction from the melt to the seed, seed-holder etc and ultimately to the surrounding through seed-melt interface. At the interface, latent heat of fusion is generated due to freezing of a part of the melt. In addition, the melt loses heat by radiation and by convection and conduction to the 
surrounding medium. By suitable choice of the material of the seed holder, a controllable method of cooling it and by controlling the ambient atmosphere, the dissipation of heat is controlled. The axis of the thermal system and the mechanical pulling and rotation system should coincide to avoid thermally-induced strains.

The shape and structure of the interface is sensitive to the perfection and composition of the growing crystal. All efforts are made to eliminate disturbances to these by mechanical vibrations. The pulling and the rotary motions of the seed are performed with the help of the electrical motors. These generate mechanical vibrations. These vibrations are isolated and not allowed to reach the surroundings. Also, there are vibrations in the surroundings which are not allowed to reach the crystal-melt interface.

Due to these considerations an apparatus for growth of crystals by the Czochralski method is required to perform the basic functions: (a) heating the starting material in a suitable crucible to the molten state and controlling the temperature of the melt; (b) providing a seed-holder assembly which can be rotated and lifted at desired rates in a smooth and uniform manner; (c) controlling the environment of the crystal growth system; (d) isolating mechanical vibrations generated in the system and in the surroundings from the crystal-melt interface; and (e) the axis of the crystal pulling and rotation, the axis of the crucible rotation and translation and the axis of the thermal system should coincide.

For details of the crystal growth techniques reference is made to recent publications (Paorici 1982; Pamplin 1975; Bardsley et al 1979) and the literature cited therein.

\subsection{The crystal pulling system developed at the National Physical Laboratory}

Figure 2 shows a photograph of the crystal growth system developed at the NPL (Krishan Lal and Singh 1977; Krishan Lal 1981, 1982). The scattering is mainly due to provided by a screw and nut assembly. The seed holder is fixed to the nut and the screw is held at its position and executes only the rotary motion. The nut moves up and down depending upon the sense of rotation of the screw. The screw is a component made to have a uniform pitch along its length of nearly $80 \mathrm{~cm}$.

The nut is also prepared with care and is cut into two equal halves parallel to the axis of threads. The two parts are held together with the help of spring loaded screws. The pulling motion is guided by two steel rods. One of these has a circular cross-section whereas the other has a square cross-section. The surface of the rod with circular crosssection has been lapped after careful machining to ensure uniformity in diameter and straightness. The other rod has been prepared in the same way as surface plates are made. Its surfaces are flat within $5 \mu$ m over its entire length of nearly $80 \mathrm{~cm}$. The screw is driven with the help of a variable speed motor and a gear box. The rate of pulling can be varied by a factor of 10 in the range $2 \mathrm{~mm} / \mathrm{hr}$ to $20 \mathrm{~mm} / \mathrm{hr}$. By changing the gear box, it is possible to have another set of pulling rates varying in a range $1: 10$. The variation in the speed is achieved steplessly with the help of electronic control of the motor. The total length of pulling is about $60 \mathrm{~cm}$.

The seed-holder is fixed to a subassembly fixed to the nut of pulling assembly. On this subassembly a $12 \mathrm{~W}$ synchronous motor is mounted which provides the motion to rotate the seed-holder. The speed of rotation can be varied to desired value. The seed holders were made out of stainless steel, silver plated copper or silver plated brass rods. Between the seed-holder and the sub-assembly on the nut a ceramic rod made of 


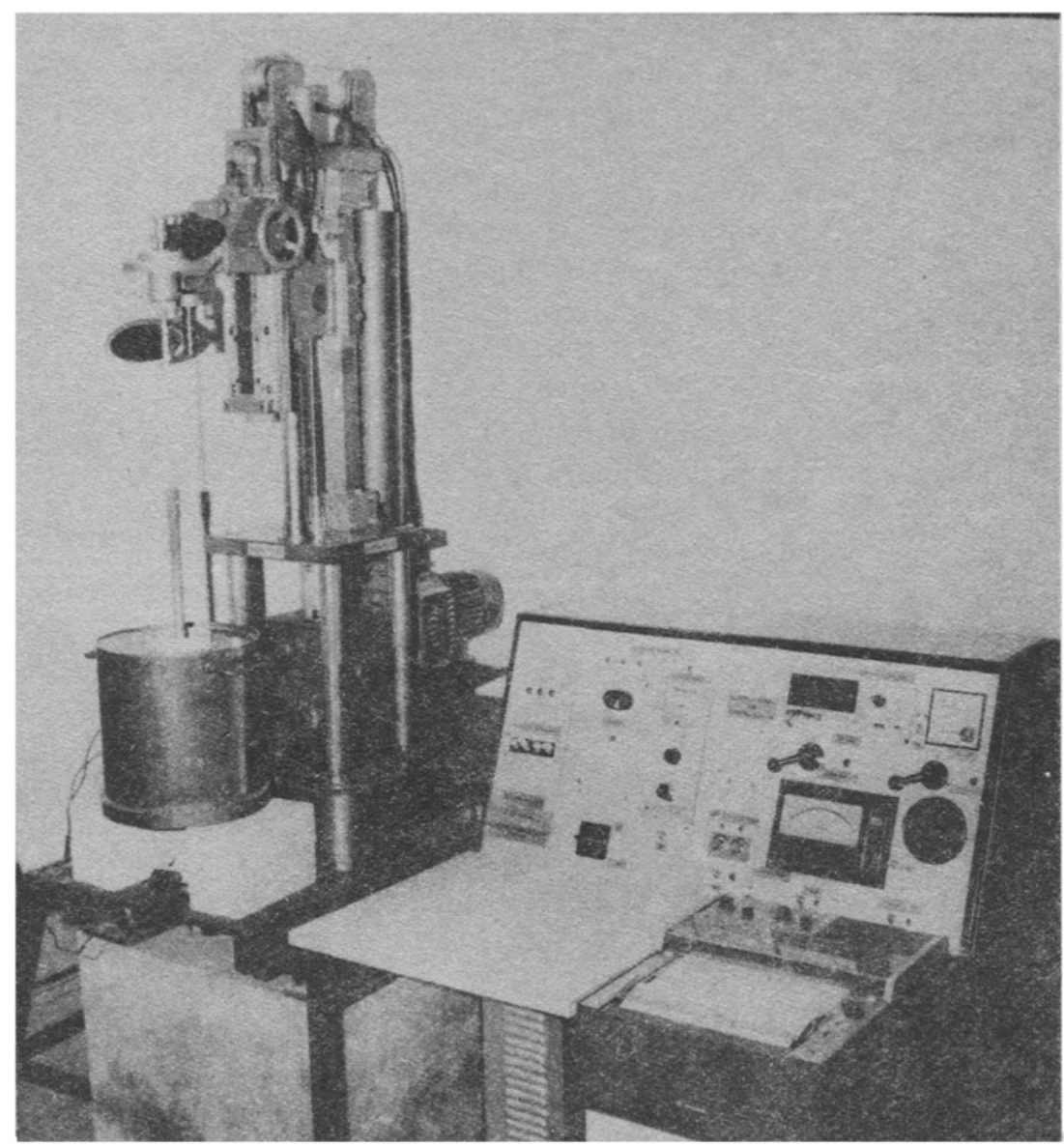

Figure 2. A photograph of the crystal. Pulling system developed at the National Physical Laboratory, New Delhi

alumina provides the thermal insulation. The seed-holder geometry has been optimized for controlled heat dissipation during crystal growth experiment.

The screw and the guiding rods are held at place with the help of thick steel plates, one each at the two ends. The assembly is fixed on a heavy base plate. To isolate the system from the vibrations in the surroundings, the base plate rests on four antivibration mountings supplied by $\mathrm{M} / \mathrm{s}$ Dunlop. The mountings lie on a block prepared out of cement and concrete. The motor and the gear box used to drive the screw for pulling motion are mounted on a separate platform. This platform rests on small antivibration mountings fixed on the base plate. The couplings between the motor and the gear box and the gearbox and the screw are flexible. These arrangements ensure that the vibrations generated by the motor are damped to a low level.

Resistance-heated furnaces have been used for crystal growth experiments. The furnace is placed on a platform rigid connected to the base of the crystal puller. The axis of the furnace, the line of pulling and the axis of rotation of the seed holder coincide with each other. 


\subsection{Some typical results}

The crystal growth system described above has been used to grow nearly perfect crystals of alkali halides (Krishan Lal et al 1982; Verma 1982). Besides optimization of the thermal system and testing of the equipment this experiment had another purpose also. In a recent investigation we had observed that in silicon single crystals, the elastic thermal waves or phonons do not contribute significantly to the diffuse $\mathrm{x}$-ray scattering (Krishan Lal and Singh 1977; Krishan Lal 1981, 1982). The scattering is mainly due to defects. We shall consider this aspect later in this paper in some detail. Since silicon has a high Debye temperature it was considered desirable to perform experiments on crystals with lower Debye temperature. Alkali halides, particularly $\mathrm{KCl}$ and $\mathrm{KBr}$ not only fulfil this requirement, but have been studied thoroughly in the past (Ramachandran and Wooster 1951; Wooster 1962). However, for diffuse scattering measurements at high resolution nearly perfect crystals are required. Hence, growth of nearly perfect $\mathrm{KCl}, \mathrm{KBr}$ and $\mathrm{NaCl}$ crystals was undertaken. Alkali halide crystals grown by the usual methods are known to possess a low degree of perfection (Krishan Lal 1980; Krishan Lal et al 1979).

- By controlling (i) the quality of the seed-crystal; (ii) the thermal conditions during crystal growth; and (iii) the rate of pulling during growth. nearly perfect alkali halide crystals have been grown (Krishan Lal et al 1982).

The maximum diameter of the crystals was about $60 \mathrm{~mm}$. Figure 3 shows some of the crystals grown on this system.

The alkali halide crystals were thoroughly characterized regarding perfection by using $x$-ray diffraction topography and multicrystal $x$-ray diffractometry. These systems are described later in this paper. Figure 4 shows a typical topograph of a $\mathrm{KCl}$ crystal. The perfection of these crystals was so high that dislocations could be easily resolved in their topographs. These have been characterized regarding their nature.

Figure 5 shows a typical diffraction curve of a $\mathrm{KCl}$ single crystal recorded on the triple crystal $x$-ray diffractometer. This curve is fairly sharp. The half width of diffraction curves of different alkali halide crystals was found to be in the range $10-30 \mathrm{sec}$ of arc. This is not very large compared to that observed with dislocation free

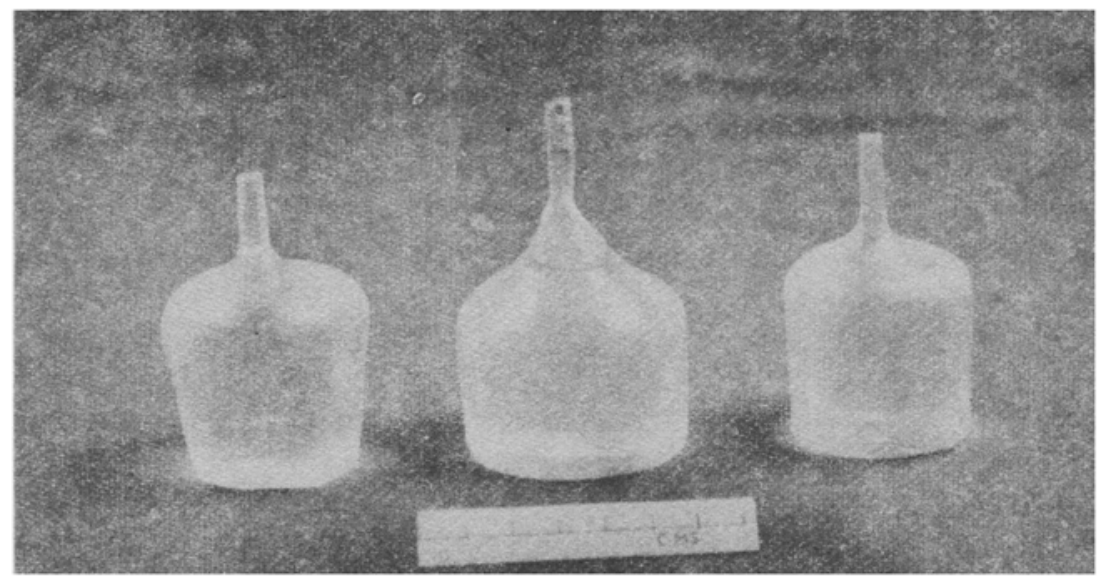

Figure 3. A photograph of a few nearly perfect alkali halide crystals grown on the crystal puller develoned at the National Physical Laboratory, New Delhi 


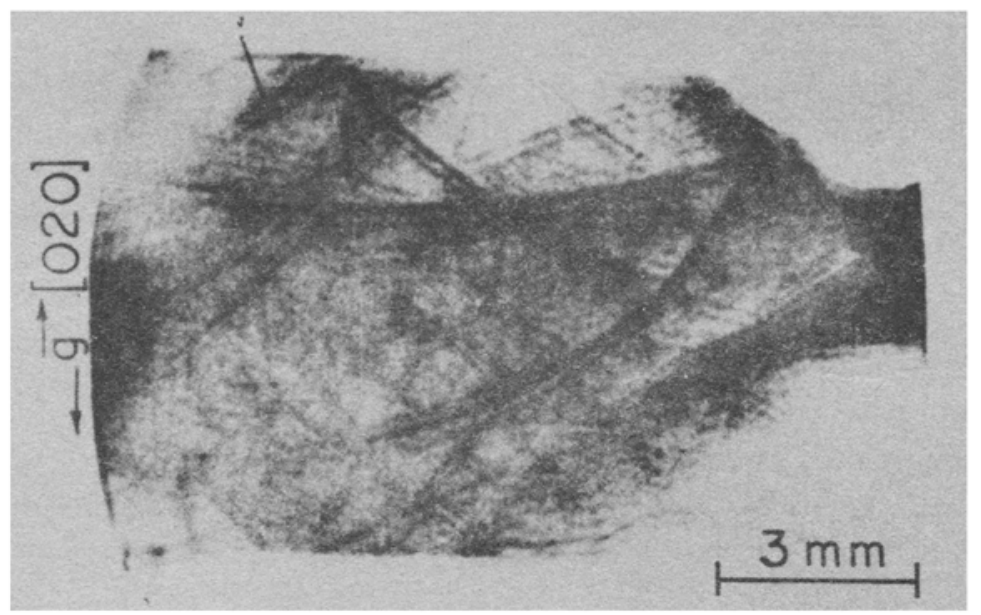

Figure 4. A typical projection topograph of a single crystal of potassium chloride grown at the NPL. 020 RELP and MoK $\alpha_{1}$ radiation was used.

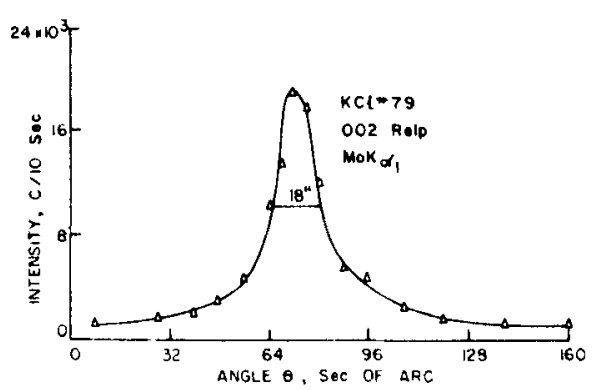

Figure 5. A typical diffraction curve of a nearly perfect single crystal of $\mathrm{KCl}$ grown at the NPL, recorded on the triple crystal $x$-ray diffractometer, in $(+,-,+)$ configuration with $\operatorname{MoK} \alpha_{1}$ radiation and $[002]$ reciprocal lattice vector

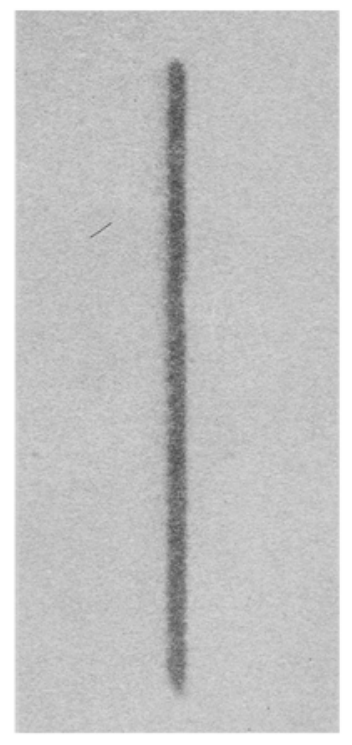

Figure 6. A high resolution topograph recorded on the triple crystal $x$-ray diffactometer, by aligning the specimen $\mathrm{KCl}$ single crystal at the peak of the diffraction curve shown in figure 5 . 


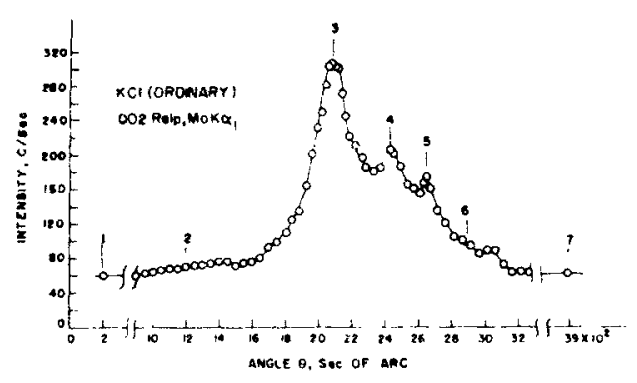

Figure 7. A typical diffraction curve of a potassium chloride single crystal of ordinary perfection recorded on the triple crystal $x$-ray diffractometer

Si single crystals ( $5 \mathrm{sec}$ of arc for 111 reflection recorded under similar conditions). High resolution small area topographs can be obtained by recording the diffracted $x$-ray beam on a film. Figure 6 shows a typical topograph of this type recorded by aligning the crystal at the peak of the diffraction curve shown in figure 5. Uniform distribution of intensity is to be noted.

Crystals having ordinary degree of perfection give diffraction curves spread over large angular range of a degree of arc or so. Figure 7 shows a diffraction curve of one such crystal recorded under the conditions under which the curve in figure 5 was recorded. The high resolution topographs of such crystals are fragmented into small regions.

\section{Equipment for characterization of single crystals regarding perfection by using high resolution $x$-ray diffraction techniques}

\subsection{Basic principles}

$\mathrm{X}$-ray diffraction has been extensively used for determination of structure of crystals right from the time of its discovery. The structure obtained in these experiments is based on absolutely ideal periodic arrangement of the constituent units. As mentioned above, the real crystals always contain lattice imperfections or defects. X-ray diffraction methods are also used to study defects. X-ray Laue method could be considered to be the first in a series of methods. The resolution attainable in the Laue method is comparable to that of the other $\mathrm{x}$-ray diffraction methods. It is convenient to define the resolution of the diffraction techniques in terms of: (i) the divergence of exploring $x$-ray beam in the plane of diffraction which is usually the horizontal plane; (ii) the monochromaticity of exploring $x$-ray beam; and (iii) the degree of perfection of the specimen crystal.

In most of the standard $x$-ray diffraction methods the divergence of the exploring beam is of the order of a degree of arc in the horizontal plane. Filtered or crystal monochromated $\mathrm{K} \alpha$ beams are used. Nearly perfect crystals have become available after development of techniques for growth of nearly perfect semiconducting crystals. However, a nearly perfect crystal will diffract over an angular range of a degree of arc when a standard diffraction system is used. As we shall see below this is nearly three orders of magnitude larger than the theoretically expected angular range under ideal experimental conditions. It may be mentioned that such a resolution is good enough for determination of crystal structure. However, when the purpose of the experiment is to observe and characterize defects such a resolution is inadequate. 
Let us now consider an ideal experiment in which a perfectly parallel and monochromatic beam of $x$-rays is diffracted from an absolutely perfect crystal. According to dynamical theory (Batterman and Cole 1964; Tanner 1976) of x-ray diffraction, $x$-rays will be diffracted over a narrow angular range of a few seconds of arc in such an experiment. The half width of the diffraction curve width is given by

$$
\theta_{1 / 2}=\frac{2 r_{e} \lambda C\left(F_{\bar{g}} F_{q}\right)^{1 / 2}}{\pi V_{c}|g| \cos \theta_{B}}
$$

where $r_{e}=$ classical electron radius, $\lambda=$ wavelength of the exploring $\mathrm{x}$-ray beam, $C=$ polarization factor, $F_{g}=$ structure factor for diffraction vector $g, \mathbf{g}=$ diffraction vector, $V_{c}=$ volume of the unit cell. Hence, it is seen that the standard x-ray diffraction experiments cannot be used for attaining the ideal curves. For this the monochromatization of the exploring $\mathrm{x}$-ray beam has to be considerably improved. This high resolution is necessary if departures from ideal arrangement of atoms are to be observed and characterized. In the following, we shall describe two major equipments, namely: (i) an $\mathrm{x}$-ray diffraction topographic system (ii) a triple crystal $\mathrm{x}$-ray diffractometer.

In the first system, the divergence of the $x$-ray beam is reduced to less than a min of arc or so in the horizontal plane. The collimation and the monochromaticity of the exploring $\mathrm{x}$-ray beam in the second system is the best achieved so far.

3.2 Equipment for $x$-ray diffraction topography: $A$ microfocus $x$-ray generator and an $x$-ray diffraction topography camera

3.2a Basic principle of $x$-ray diffraction topography. The basic principle of $x$-ray diffraction topography is that the intensity of diffracted $x$-rays depends upon the degree of perfection of the irradiated volume for a given reflection and $\mathrm{x}$-ray wavelength. Hence, a point-to-point variation of diffracted $x$-ray intensity from a specimen gives information about distribution of defects in the crystal. If we are able to record diffracted intensity from the entire volume of a specimen in such a manner that each point in the recorded pattern corresponds to a point in the specimen, the defects will be imaged directly in this record. Such a record is known as topograph and is essentially a Laue spot of the entire specimen. The first experiments in this direction were performed by Ramachandran (1944). A number of different approaches have been adopted to record the intensity variations in the topographs with sufficiently high resolution (Krishan Lal 1982; Tanner 1976; Barret 1945; Lang 1970).

For a good correlation between the point-to-point variation of intensity in the topograph and the corresponding variation of perfection in the specimen, the following experimental conditions should be fulfilled (for details see Krishan Lal 1982): i.e. (1) The size of the source should be as small as possible; (2) the distance between the source and the specimen should be as large as possible; and (3) the photographic film/plate should be placed very close to the specimen.

Figure 8 shows a schematic line diagram of a typical experimental arrangement which fulfils the three conditions listed above. It can be seen that the irradiated area of the specimen is ribbon shaped, its length being perpendicular to the plane of the figure. Since the width is very small (typically $100 \mu \mathrm{m}$ or less), at a time only a small volume of the crystal can be investigated. Lang introduced the technique of moving the specimen crystal across the x-ray beam (Lang 1970). The photographic film or plate is rigidly coupled with specimen and the two together are traversed across the exploring beam. In 


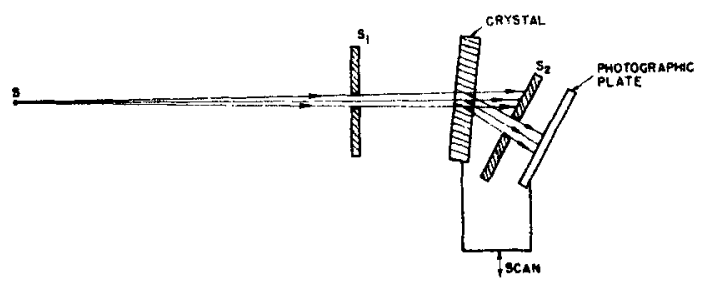

Figure 8. A schematic line diagram of the x-ray diffraction topographic set-up.

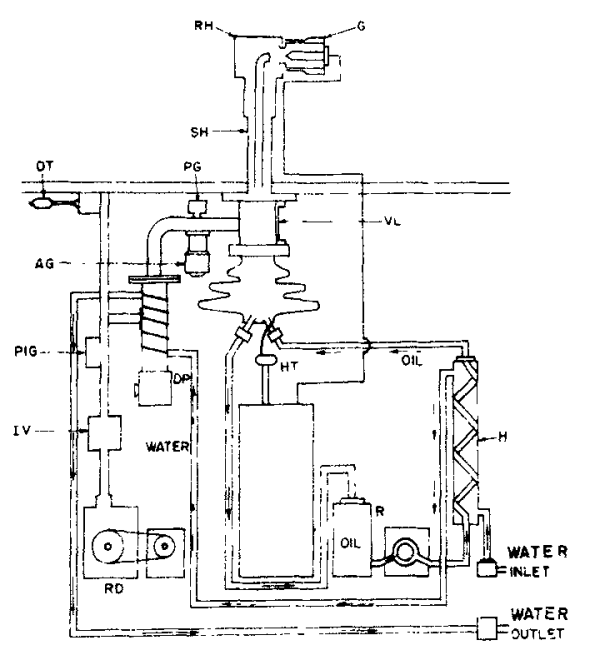

Figure 9. A schematic line diagram of the microfocus $\mathrm{X}$-ray generator developed at the NPL.

this manner crystals with very large volumes (several $\mathrm{cm}^{3}$ ) can be investigated. This technique is known as the Lang method.

This method can be used for observation and characterization of grain boundaries, low angle boundaries and dislocations. In the following we shall describe a system developed at the NPL which consists of two major equipment: (i) a microfocus X-ray generator; and (ii) an x-ray diffraction topography camera.

3.2b The microfocus x-ray generator developed at the NPL: Figure 9 shows a schematic line diagram of the microfocus $x$-ray generator (Krishan Lal et al 1975). Figure 10 is a photograph of this equipment. A hairpin filament fixed in a specially designed electron gun is heated to be a source of electrons. The electrons are accelerated and focussed electrostatically with a bias cup on the surface of the anode. The distance between the surface of the anode and the source of electrons can be varied continuously to optimize the focussing conditions. The path of the electrons is evacuated to be $\sim 10^{-5}$ torr.

Filaments of desired shapes are prepared from thin tungsten wires with the help of special jigs. These are held in supports which are precisely aligned. The filament terminals are connected to insulating feedthroughs which can stand high temperatures and high vacuum. The feedthroughs have been developed in our laboratory.

The bias cup is a precisely made component whose position is fixed accurately with respect to the filament. These components are fixed into the inner part of the electron gun which also has an outer part. Both the parts are made of stainless steel. The filament together with the bias cup can be linearly moved towards or away from the anode when 


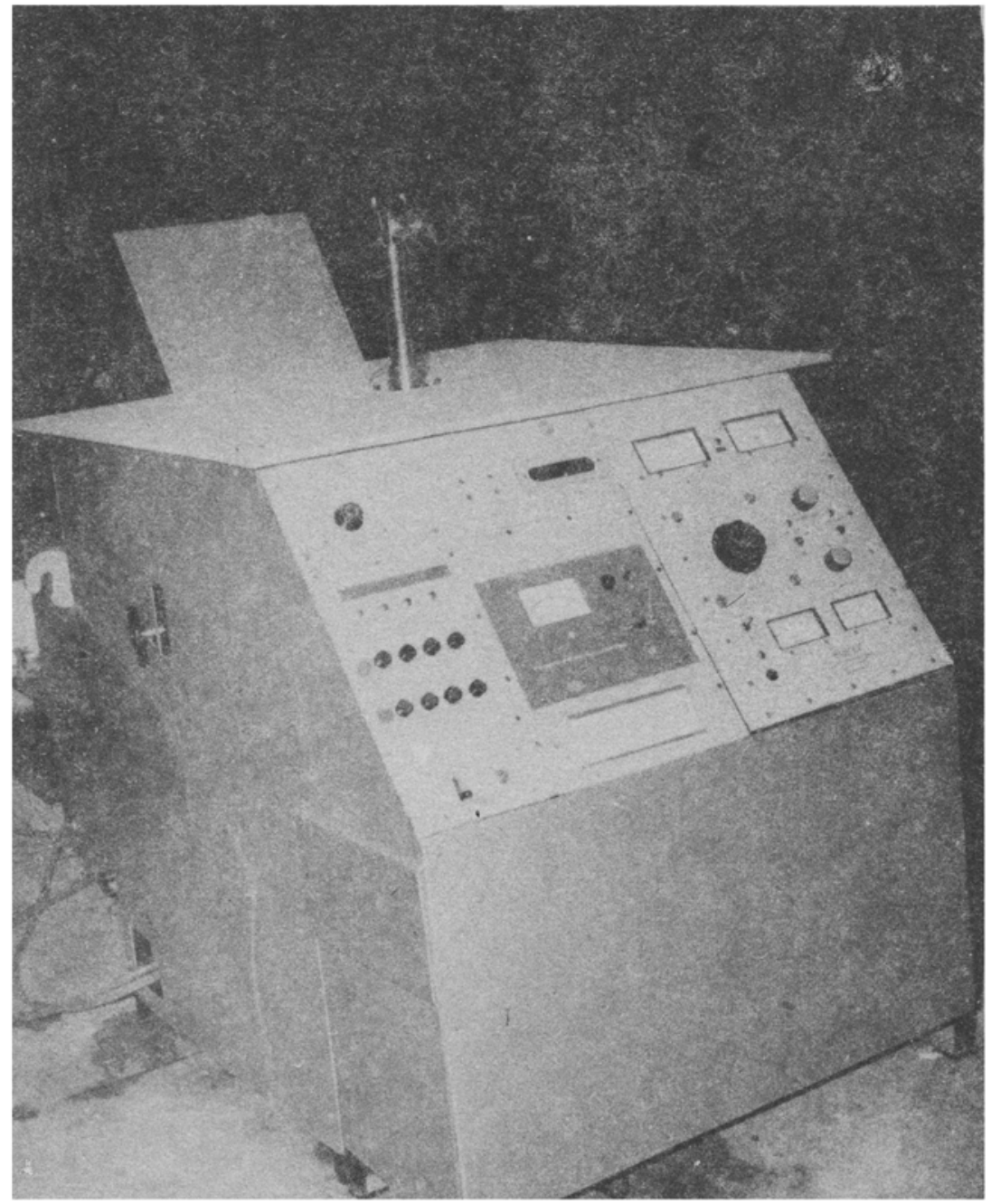

Figure 10. A photograph of the microfocus $x$-ray generator developed at the NPL.

the generator is in operation. The electron gun after assembly is fixed to the body of the vacuum chamber which also houses the anode.

The anode is a hollow pipe fixed into an insulating epoxy block. Its upper open end is bent by $90^{\circ}$ and a thin disc of the target material is welded. (Inside this pipe another small pipe is inserted, which is all along its length and has a small opening in its end just behind the target.) Chilled oil forced through the smaller pipe which hits the back surface of the target and flows to the reservoir through the space between the two pipes. This is the coolant during $\mathrm{x}$-ray generation. In the standard sealed tubes, the targets are cooled by chilled water. However, unlike in this case, in the microfocus generator the anode is at the high positive potential and therefore, water cannot be used as coolant. An oil with good thermal conduction and high insulation strength is used. The high voltage is applied to terminal moulded in the epoxy base. 
The electron gun and the anode are fixed to a metal chamber made precisely. It is evacuated to a vacuum of about $10^{-5}$ torr with the help of an oil diffusion pump backed by a rotary pump. The openings for the electron gun and the anode are made precisely, so that the axis of the electron gun is perpendicular to the surface of the anode. The vacuum in the chamber is continuously monitored. Also the vacuum generated by the backing pump is continuously monitored.

The focal spot on the anode is $40 \mu \mathrm{m}$ in diameter when the generator is used as a point source. This system can also be used in the line focus mode, with the width of the line as $100 \mu \mathrm{m}$ and length as $\sim 2 \mathrm{~mm}$. The electrical power consumed by the generator is $100 \mathrm{~W}$ in line focus mode and only $10 \mathrm{~W}$ in the point focus mode. These powers are very small compared to the powers consumed by the sealed tubes $(1-2 \mathrm{~kW})$. However, it may be emphasized that in the high resolution experiments, the brilliance of the source is more important. It is defined as the power dissipated per unit area of the source on the anode. The brilliance of the point focus generator is about $8 \mathrm{~kW} / \mathrm{mm}^{2}$ when the power is only $10 \mathrm{~W}$. The standard focal size sealed tubes $\left(1 \times 10 \mathrm{~mm}^{2}\right)$ have a brilliance of $200 \mathrm{~W} / \mathrm{mm}^{2}$ when run at the full power of $2 \mathrm{~kW}$. Recently, fine focus sealed tubes have become available with powers of $\sim 2 \mathrm{~kW}$.

3.2c. The X-ray diffraction topography camera developed at the NPL: Figure 11 is a schematic line diagram of the $x$-ray diffraction topography camera developed at the NPL. A photograph of this system is shown in figure 12 (Krishan Lal et al 1974; Krishan Lal 1982). X-ray beam from a microfocus $x$-ray source is collimated with the help of a long collimator tube which has a narrow vertical slit at its end away from the source. The tube is $50 \mathrm{~cm}$ long, the width of the slit is variable. It is about $100 \mu \mathrm{m}$ for projection topography. The divergence of the beam can be reduced to less than a min of arc in the horizontal plane (the plane of the figure). A horizontal slit is also generally introduced with the horizontal slit to limit the dimensions of the exploring beam in the vertical direction. The size is governed by the dimensions of the specimen in the vertical direction.

The specimen to be investigated is in the form of a disc which is mounted in a vertical circle goniometer with its larger surfaces in the plane of the goniometer circle. The goniometer provides rotations to the specimen around a horizontal axis which is perpendicular to the surface of the specimen. The goniometer is mounted on a traversing mechanism which in turn is mounted on top of a turntable. The turntable provides rotations to the specimen around a vertical axis passing through its middle. The traversing mechanism provides to- and fro- motion to the specimen across the $x$ ray beam. A scintillation counter is used as $x$-ray detector. It is mounted on the turntable with its axis along a radius of the turntable and in the horizontal plane containing the axis of the collimator. The detector can be rotated around the vertical

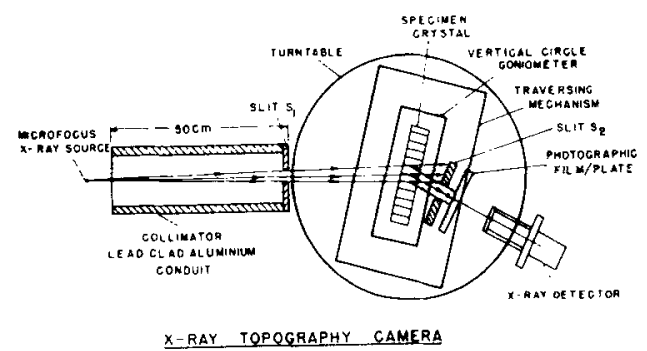

Figure 11. A schematic line diagram of the $x$-ray diffraction topography camera developed at the NPL. 


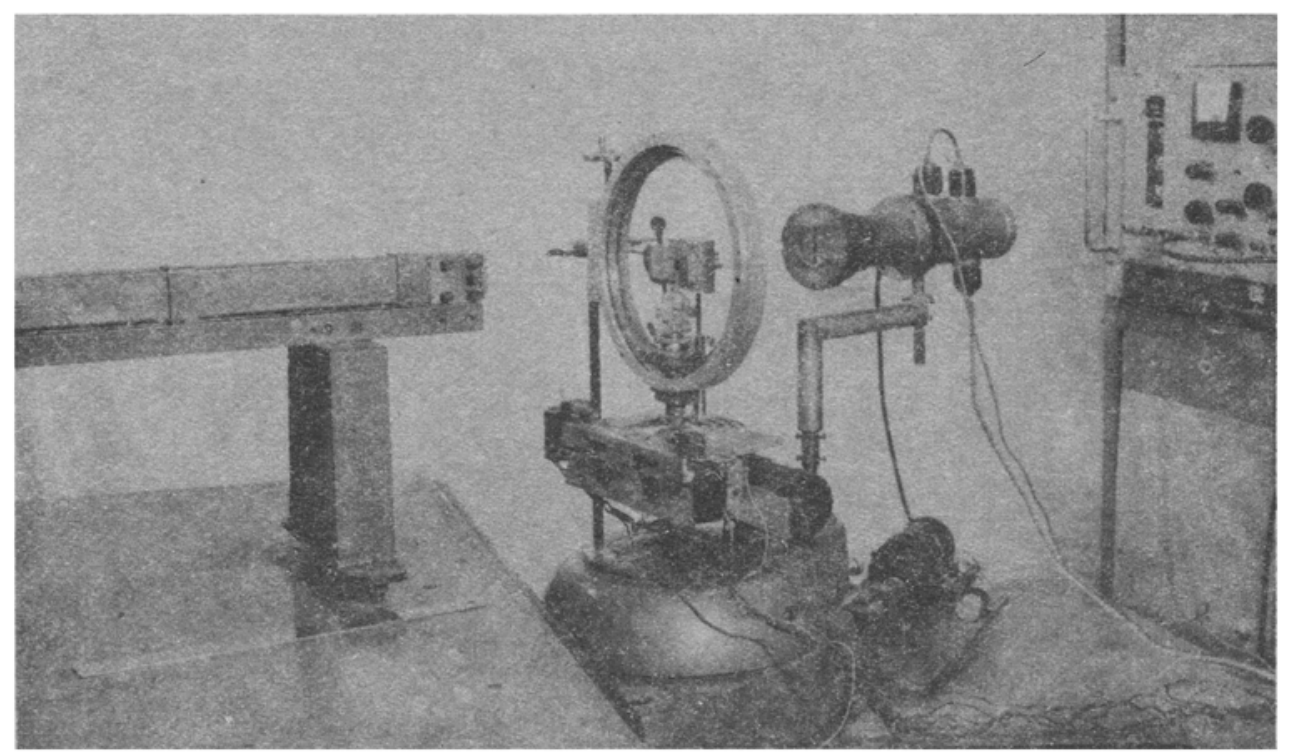

Figure 12. A photograph of the $\mathrm{x}$-ray diffraction topography camera developed at the NPL.

axis of the turntable around which the specimen is also rotated. The angular positions of both can be read on the turntable. The mechanical precision is required to ensure that the axis of the collimator is horizontal and intersects the axis of the turntable.

The photographic film or plate is fixed rigidly on top of the traversing mechanism and very close to the specimen. A vertical slit is introduced between the specimen and the film/plate. Its position is adjusted to allow only the diffracted beam to pass through and to stop the direct beam. This slit is rigidly coupled to the turntable.

The turntable is required to provide small angular movements of a few sec of arc to the specimen. A long radial arm $(50 \mathrm{~cm})$ is attached to the axis of the turntable. The end of this arm is moved with the help of a micrometer screw. The arm can be decoupled whenever larger angular movements are to be given to the crystal. The angular movements of the detector are not required that precisely and therefore this can be accomplished without radial arm.

The traversing mechanism is also a component requiring careful design and fabrication. It is required to provide very smooth and uniform motion to the specimen and the photographic film/plate. It consists of two parts: (i) a base plate and (ii) a moving platform. The two are coupled to each other with the help of a screw and nut assembly. The screw is fixed in its position on top of the base plate with its axis along the longer edge of the plate. The nut is rigidly coupled to the bottom of the moving platform. When the screw is rotated the upper plate moves linearly due to the motion of the nut. This movement is guided with the help of a rod of square cross-section and a flat plate. These are fixed, one each, along the two longer edges of the base plate. A steel ball rolls loosely on the flat plate. The upper plate has a flat strip which rests on the ball when the traversing mechanism is assembled. The other edge of the top plate has a groove which fits the rod of square cross-section fixed on the base plate. These two, the guiding rod and the plate, ensure a smooth and uniform linear movement of the moving platform and thus the crystal. The micrometer screw is driven by a synchronous motor 
$(12 \mathrm{~W})$. To reduce the speed of the motor $(1500 \mathrm{rpm})$ a series of gears are introduced between the screw and the motor. The screw rotates at a speed of $5 \mathrm{rpm}$. The range of scan is adjusted electrically with the help of two microswitches fixed to the base plate and an airbreak contactor. The range of traverse equals the dimensions of the specimen along the line of traverse.

For aligning the specimen for diffraction from a desired set of lattice planes, the detector is rotated from its reference (direct beam receiving) position by an angle $2 \theta_{B}$, where $\theta_{B}$ is the Bragg angle. By using the angular movements provided by the vertical circle goniometer and the turntable, it is possible to align the specimen for diffraction from any desired set of lattice planes.

3.2d Some typical 'results: Before recording topographs of the specimen, it is advantageous to first record a diffraction curve or a rocking curve. This curve is obtained by plotting diffracted intensity as a function of the grazing angle covering angular range on both sides of the diffraction peak. The shape of the diffraction curve is very sensitive to the degree of perfection of the specimen crystal. Nearly perfect crystals give well resolved diffraction maxima due to $K \alpha_{1}$ and $K \alpha_{2}$ components of the characteristic radiation. The half width of each peak is dependent on the divergence of the exploring $\mathrm{x}$-ray beam. Figure 13 shows a typical diffraction curve of a dislocation free silicon single crystal.

Specimen crystals containing boundaries give diffraction curves having several peaks separated by large angles. When low angle boundaries are the main defects, the separation between various peaks is of a few min of arc. In the case of grain boundaries the various peaks in the diffraction curve are separated by about a degree of arc. Figure 14 shows a typical diffraction curve of a crystal containing low angle boundaries. It may be remarked that $x$-ray topography is profitably used to characterize crystals which are free of boundaries. Low angle and grain boundaries can be conveniently studied by $\mathrm{x}$ ray Laue method (Krishan Lal et al 1970, 1972).

Figure 15 shows a topograph of a nearly perfect single crystal of $\alpha-\mathrm{Al}_{2} \mathrm{O}_{3}$ which had been grown by the chemical vapour deposition (Krishan Lal and Kumar 1978). The

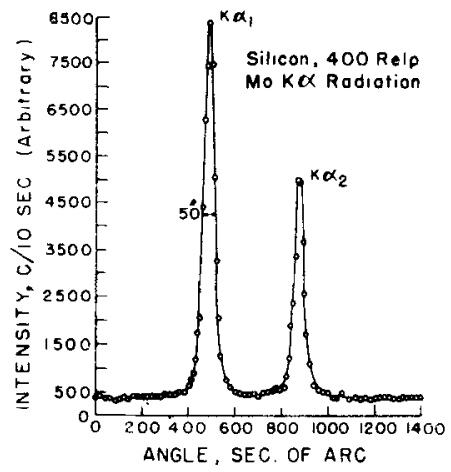

Figure 13. A typical diffraction curve of a nearly perfect crystal recorded on the $x$-ray diffraction topography camera. Wellresolved peaks due to $K \alpha_{1}$ and $K \alpha_{2}$ comporients of the characteristic $K \alpha$ line are observed.

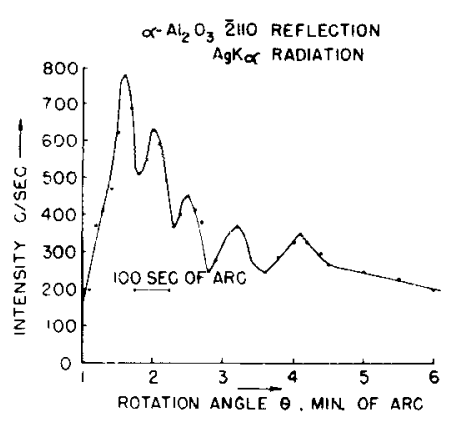

Figure 14. A typical diffraction curve of a crystal containing low angle boundaries. Each peak in this curve corresponds to diffraction from a subgrain of the crystal. 


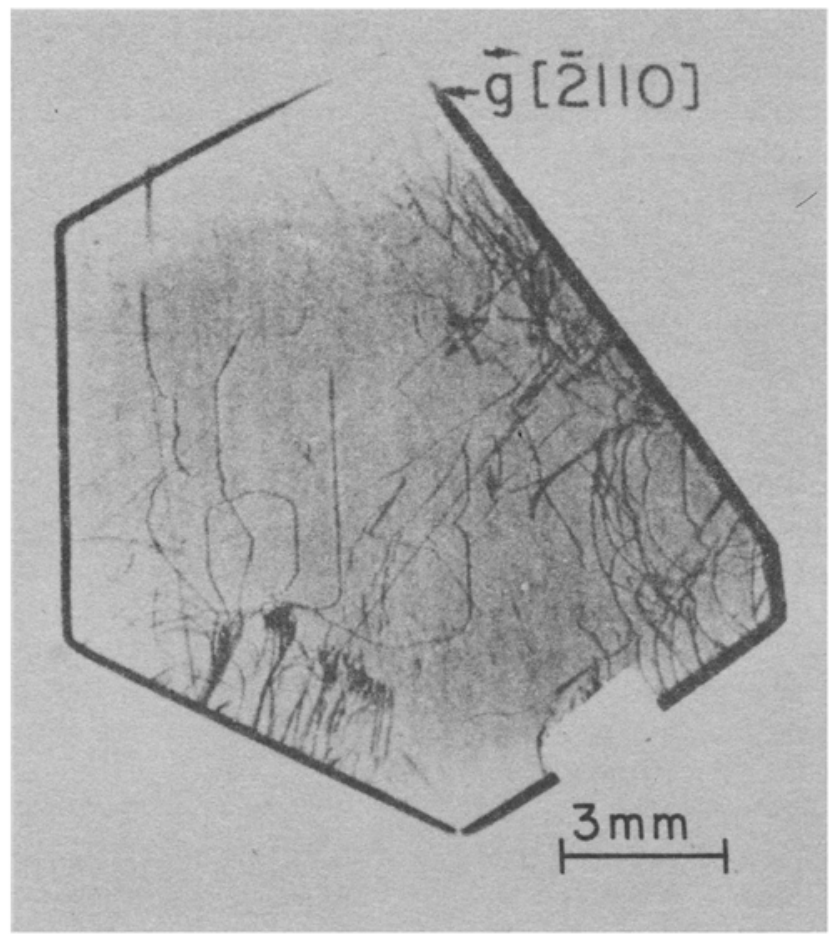

Figure 15. A typical projection topograph of a nearly perfect $\alpha-\mathrm{Al}_{2} \mathrm{O}_{3}$ single crystal grown by the chemical vapour deposition method. MoK $\alpha_{1}$ radiation and $\left[\begin{array}{lll}2 & 1 & 0\end{array}\right]$ diffraction vector was used

lines observed in this topograph are dislocation lines. With this method it is possible not only to observe the dislocations but also to characterize these for their nature (Krishan Lal 1982). This is possible as the strain around dislocations is anisotropic. Maximum strain is produced in lattice planes which are perpendicular to the Burgers vector of the dislocation. The contrast around dislocation images in the topographs depends directly on the strain produced by dislocations in the diffracting planes. Hence by recording topographs with different diffracting planes, the nature of dislocations can be determined from the observed variations in contrast around dislocation images. For details, reference is made to published literature (Krishan Lal 1982; Tanner 1976; Lang 1970).

\subsection{The triple crystal $x$-ray diffractometer developed at NPL}

3.3a Background. Chemical composition and the real structure of crystals govern their physical properties. It has been recently realized that the properties of dislocation free crystals having nominally the same chemical composition are different. For example, the electronic properties of dislocation free single crystals vary significantly (Yang et al 1978; Yang and Schwuttke 1980). These differences are attributed to point defects and their aggregates. Hence, characterization of dislocation-free crystals regarding perfection, requires information about point defects and their clusters. These defects do not produce sufficient contrast in topographs and it is not possible to use $\mathrm{x}$-ray diffraction 
topography for this purpose. However, point defect aggregates are known to produce scattering from reciprocal space near a reciprocal lattice point (Krishan Lal 1980, 1982; Krivoglaz 1969; Dederichs 1973; Peisl 1976). This is termed as the diffuse x-ray scattering or DXS. DXs is also produced by phonons as mentioned above (Ramachandran and Wooster 1951; Wooster 1962).

It was of considerable fundamental importance to establish the relative magnitudes of the Dxs due to defects and phonons. In the earlier investigations Dxs was considered to be due entirely to the phonons (Wooster 1962). Our preliminary experiments even with an x-ray diffraction topographic equipment showed that this is not so (Krishan Lal and Singh 1977; Krishan Lal 1978). In the earlier experiments Dxs measurements were made with low resolution experimental set-ups. However, it needs to be stressed that whenever scattering is to be measured near the reciprocal lattice point the resolution of the method becomes all the more important. In view of these considerations, we have designed, developed and fabricated a triple crystal $x$-ray diffractometer in which the experimental uncertainties have been reduced to a negligible level. Further, the same diffractometer can also be used to record diffraction curves and high resolution small area topographs. This system is described in the following.

3.3b The triple crystal $x$-ray diffractometer: Figures 16 and 17 , respectively, show a schematic line diagram and a photograph of the diffractometer. A microfocus $x$-ray generator with a focal spot size of $40 \mu \mathrm{m}$ size on the anode is used as the source of $\mathrm{x}$ rays. The $x$-ray beam is collimated, as in the case of the topographic system, to have a divergence of about $1 \mathrm{~min}$ of arc in the horizontal plane. This beam is diffracted from a set of two monochromator crystals of the Bonse-Hart type (Bonse and Hart 1965). These are prepared out of a dislocation free Si single crystal block and have their diffracting surfaces parallel to $\{111\}$ in $(+,-)$ configuration. On alignment for diffraction, these monochromators are capable of resolving the $K \alpha_{1}$ and $K \alpha_{2}$ components of the $\mathrm{K} \alpha$ doublet. A slit is placed in the path of the diffracted beam which allows only the $\mathrm{K} \alpha_{1}$ beam to pass through. The wavelength, direction and spatial spreads of this beam have been experimentally evaluated (Krishan Lal 1979; Krishan Lal and Singh 1979). The divergence could not be detected in the horizontal plane as it was much less than $5 \mathrm{sec}$ of arc, the limit of detection. The wavelength spread in this beam was evaluated by using an analyzer crystal in the dispersive geometry. This was observed to be from 0.7088 ro $0.7097 \AA$. The width of the beam in the horizontal plane can be varied from $15 \mu \mathrm{m}$ to $\sim 50 \mu \mathrm{m}$. In most of the experiments a width of $\sim 50 \mu \mathrm{m}$ is used.

The specimen is mounted on a specially designed goniometer which in turn is fixed on top of a turntable. The axis of the turntable is vertical and aligned to intersect the

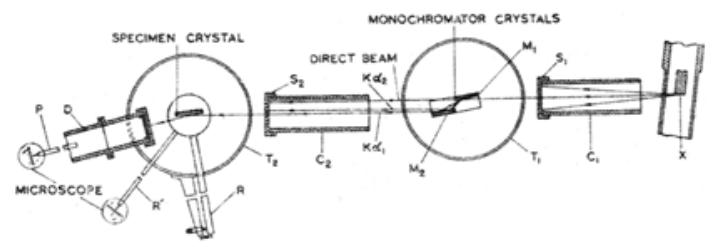

Figure 16. A schematic line diagram of the triple crystal $x$-ray diffractometer, designed, developed and fabricated at the NPL. 


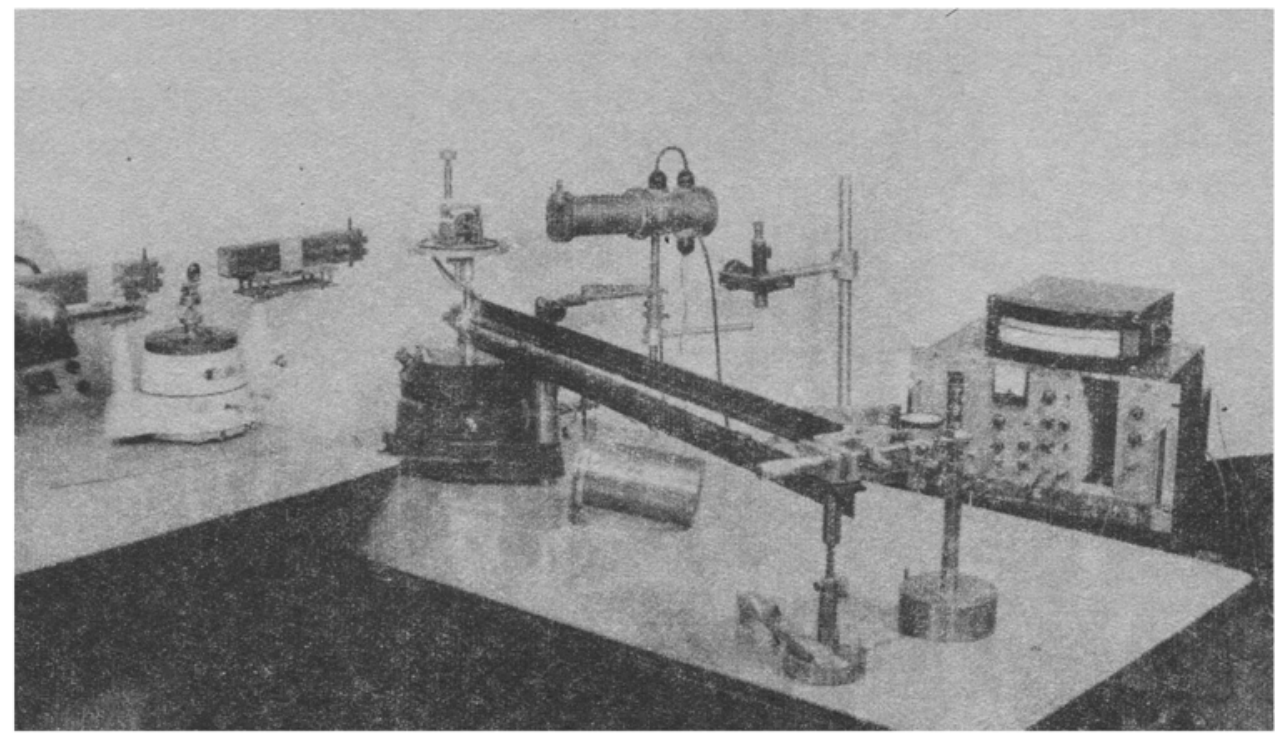

Figure 17. A photograph of the triple crystal $x$-ray diffractometer

exploring x-ray beam received from the monochromators. The position of the goniometer with the crystal, relative to the turntable is fixed in such a way that the axis of turntable lies on the surface of the specimen. The surface is parallel to the diffracting planes. The goniometer is capable of giving small linear movements to the specimen in the horizontal plane along a direction which is parallel to the diffraction vector. It can provide rotations around a horizontal axis parallel to the diffraction vector. Minimum rotation of $\sim 100 \mathrm{sec}$ of arc can be provided. For high temperature measurements a small heater can be attached behind the specimen. Two thermocouples can be attached one on the front surface of the specimen and other on the back surface of the specimen.

The turntable is required to provide very small angular rotations to the crystal. A $1 \mathrm{~m}$ long arm is attached to the turntable along a radius. The free end of the arm rests against a micrometer under tension of a spring. The fixed micrometer rests against a differential micrometer. In this way very small angular movements of about $0.3 \mathrm{sec}$ of arc can be provided to the specimen. The angular position of the specimen is measured independently by measuring the displacement of a pointer under a microscope. The pointer is attached at the end of $\mathfrak{a} 1 \mathrm{~m}$ long arm.

A scintillation counter developed in our laboratory has been used as an x-ray detector. It is mounted with its axis along one radius of the turntable and intersecting on the surface of the specimen at the same point where the axis of the exploring $x$-ray beam intersects the specimen. A fine vertical slit is fixed before the detector. Its width is slightly larger than that of the beam diffracted by the specimen. The angular position of the detector is also measured with the help of a microscope.

The diffractometer is generally used in the Bragg geometry in the configuration $(+$, $-,+)$. For measurement of diffuse x-ray scattering the specimen and the detector are rotated with respect to the position of the diffraction maximum by known angles. In this way reciprocal space in different directions can be explored. 
3.3c Some typical results: Figure 18 shows a typical diffraction curve of a dislocation free silicon single crystal recorded on the triple crystal x-ray diffractometer (Krishan Lal et al 1979). This curve is very sharp and has a half width of five sec of arc only. The shape of the diffraction curve is nearly the same as expected theoretically on the basis of the dynamical theory. Hence, we see that the reciprocal lattice point (RELP) can be very accurately located. The uncertainties due to instrumental factors are negligible. It is possible to make DXS measurements very close to RELP and at very small angular intervals of a few sec of arc. This means that the reciprocal space can be explored with a high resolution. High resolution topographs are recorded by aligning the specimen at the peak of the diffraction curve and recording the diffracted beam on a film. We have already shown one such topograph in figure 6.

This system has been used for a detailed and systematic study of diffuse $x$-ray scattering from nearly perfect single crystals of silicon (Krishan Lal and Singh 1977, 1980; Krishan Lal et al 1979; Singh 1980), potassium chloride, potassium bromide (Krishan Lal and Singh 1981a) and copper (Krishan Lal and Singh 1981b). In the first part of these investigations the aim was to evaluate the relative contribution of phonons and defects to the Dxs. Fortunately, the Dxs distribution due to the two is expected to be different. In the case of thermal diffuse scattering the intensity of Dxs (Dxs I) is expected to vary as $K^{*-2}, K^{*}$ is the wavevector of the elastic thermal waves and in reciprocal space it is obtained by joining the RELP with the elemental volume of the space being explored. The intensity along $\mathbf{K}^{*}$ and along opposite direction $\mathbf{K}^{*}$ is expected to be equal. The slopes of the lines in DXSI vs $1 / K^{* 2}$ plots for $\mathbf{K}^{*}$ perpendicular to the reciprocal lattice vector $\mathbf{R}^{*}$ are expected to be more than those of the lines with $\mathbf{K}^{*}$ parallel to $\mathbf{R}^{*}$. The Dxs I is expected to depend only on the temperature of the specimen. In the case of DxS due to defects, the Dxs I can vary as $K^{*-n}$ with $n$ having different values. In the Dxs I vs $1 / K^{* 2}$ plots the slope of lines with $\mathbf{K}^{*}$ along $\mathbf{R}^{*}$ are higher than those expected for the lines with $\mathbf{K}^{*}$ perpendicular to $\mathbf{R}^{*}$. The Dxs I is not isotropic with respect to the sense of $\mathbf{K}^{*}$. We shall consider here only one plot to illustrate the main results.

Figure 19 is a DXs I vs $1 / K^{* 2}$ plot for a nearly perfect silicon single crystal (Krishan

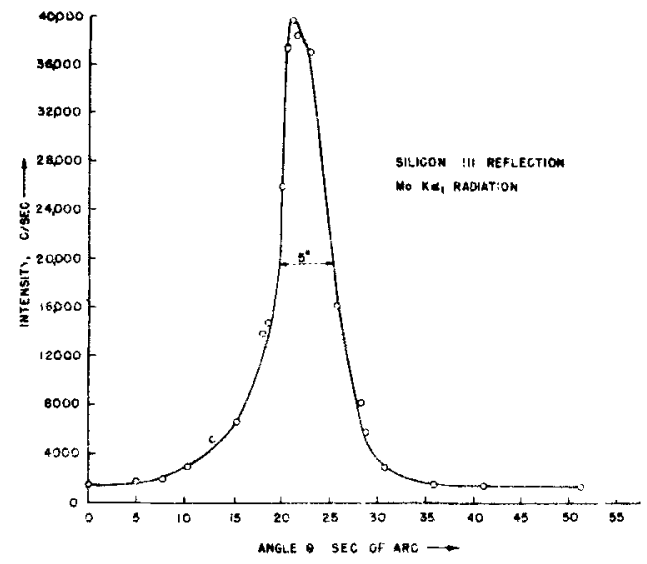

Figure 18. A typical diffraction curve of a dislocation free silicon single crystal recorded on the triple crystal $x$-ray diffractometer 111 REI.P and $\operatorname{MoK} \alpha_{1}$ radiation was used.

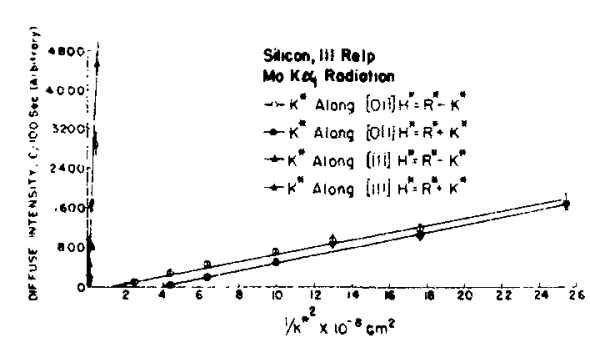

Figure 19. A typical Dxs I vs $1 / \mathrm{K}^{* 2}$ curve obtained with a dislocation free silicon single crystal. Dxs was measured around the 111 RELP with MoK $\alpha_{1}$ radiation. 
Lal and Singh 1977). It is seen that the lines with $\mathbf{K}^{*}$ parallel to $\mathbf{R}^{*}$ have higher slope than the lines with $\mathbf{K}^{*}$ perpendicular to $\mathbf{R}^{*}$. If this data is plotted on a log-log scale more than one slope is observed. This shows that the observed Dxs I is not due to elastic thermal waves.

Experiments have been performed with different specimens of $\mathrm{Si}$, with different radiations and using different RELPS and the same results were obtained. Since Debye temperature of silicon crystals is very high some experiments were performed at high temperatures. Only at $300^{\circ} \mathrm{C}$ some contribution of phonons was observed.

Experiments were performed with materials such as potassium chloride, potassium bromide and copper, having low Debye temperatures. These results also support the main conclusions.

However, it was observed that the Dxs distribution is very sensitive to the thermal history of the specimen. By analysing the Dxs data the size and shape of point defect clusters was determined in silicon. In another series of experiments, Si crystals were deliberately heated under oxygen and the oxygen aggregates were characterized by Dxs measurements. This series of investigations has led to the development of a new nondestructive tool for study of defects in dislocation-free single crystals. The results that the contribution of phonons to the Dxs is insignificant as compared to that of defects is of considerable importance.

The power of the high resolution $\mathrm{x}$-ray diffraction techniques in revealing small changes in atomic arrangement in nearly perfect crystals has been demonstrated in a series of new experiments. In these experiments, microstructural changes induced by high electric fields in single crystals of semiconductors and insulators have been directly observed and characterized for the first time (Krishan Lal and Peter Thoma 1981a, b, 1983; Peter Thoma and Krishan Lal 1982).

\section{References}

Bardsley W, Hurle D T J and Mullin J B (eds) 1979 Crystal growth: A tutorial approach (Amsterdam: NorthHolland)

Barret C S 1945 Trans. AIME 16115

Batterman B W and Cole H 1964 Rev. Mod. Phys. 36681

Bonse U and Hart M 1965 Appl. Phys. Lett. 7238

Dederichs P H 1973 J. Phys. F3 471

Krishan Lal 1978 in Advances in crystallography (ed.) R Srinivasan (New Delhi: Oxford and IBH Publishing Co.) p 136

Krishan Lai 1980 Proc. Indian Natl. Sci. Acad. A47 (Supp) 120

Krishan Lal 1981 Trans. IIM 34398

Krishan Lal 1982 in Synthesis, crystal growth and characterization (ed.) Krishan Lal (Amsterdam: NorthHolland) p 215, 287

Krishan Lal, Anand J R, Kumar H and Rawat J S 1979 Tenth National Conf. Crystallogr. Varanasi

Krishan Lal, Anantha Murthy R V, Halder S K, Singh B P and Kumar V 1982 J. Crystal Growth 56125

Krishan Lal and Jhans H K 1977 J. Phys. C10 1315

Krishan Lal and Kumar V 1978 J. Electrochem. Soc. 1252079

Krishan Lal, Kumar V and Pahwa D R 1975 National Conf. Cryst. New Delhi

Krishan Lal and Pahwa D R 1971 Rev. Sci. Instrum. 42534

Krishan Lal and Peneva S K 1968 J. Appl. Phys. 395474

Krishan Lal and Peneva S K 1969 J. Appl. Phys. 405045

Krishan Lal, Peneva S K and Verma A R 1970 Phys. Status. Solidi A3 617

Krishan Lal, Peneva S K and Verma A R 1972 Proc. Indian Natl. Sci. Acad. A38 173 
Krishan Lal and Thoma P 1981a Solid State Commun. 40637

Krishan Lal and Thoma P 1981b Acta Crystallogr. A37 C262

Krishan Lal and Thoma P 1983 Phys. Status. Solidi. A80

Krishan Lal and Singh B P 1977 Solid State Commun. 2271

Krishan Lal and Singh B P 1979 Indian J. Phys. A53 72

Krishan Lal and Singh B P 1980 Acta Crystallogr. A36 178

Krishan Lal and Singh B P 1981a J. Crystal Growth 54493

Krishan Lal and Singh B P 1981b Acta Crystallogr. A37 C-273

Krishan Lal, Singh B P and Verma A R 1979 Acta Crystallogr. A35 286

Krivoglaz M A 1969 Theory of x-ray and neutron scattering by real crystals (New York: Plenum Press)

Kumar V 1983 Ph.D. Thesis Univ. of Delhi

Lang A R 1970 in Modern diffraction and imaging techniques in material science (eds) S Amelinckx, R Gevers,

G Remant and J Landyut (Amsterdam: North-Holland) p 407

Pamplin B R (ed) 1975 Crystal Growth (New York: Pergamon Press)

Paorici C 1982 in Synthesis, crystal growth and characterization (ed.) Krishan Lal (Amsterdam: NorthHolland) p 133

Peisl H 1976 in Defects and their structure in non-metallic solids (eds) B Henderson and A E Hughes (New York and London: Plenum Press) p 381

Peneva S K 1969 Ph.D. Thesis, Univ. of Delhi, Delhi

Ramachandran G N 1944 Proc. Indian. Acad. Sci. A19 280

Ramachandran G N and Wooster W A 195: Acta Crystallogr. 4 385, 431

Singh B P 1980 Ph.D. Thesis, Univ. of Delhi, Delhi

Tanner B K 1976 X-ray diffraction topography (Oxford: Pergamon Press)

Thoma $\mathbf{P}$ and Krishan Lal 1982 in Syntheses, crystal growth and characterization (ed.) Krishan Lal (Amsterdam: North-Holland) p. 427

Verma A R 1982 in Synthesis, crystal growth and characterization (ed.) Krishan Lal (Amsterdam: NorthHolland) p 1

Verma A R, Krishan Lal, Pahwa D R, Kumar V and Aggarwal K 1974 Indian I. Pure and Appl. Phys. 12350 Wooster W A 1962 Diffuse x-ray reflections from crystals (Oxford: Clarendon Press)

Yang K H, Kappert H F and Schwuttke G H 1978 Phys. Status Solidi 50221

Yang K H and Schwuttke G H 1980 Phy's. Status. Solidi. 58127 